\title{
Triple intersection numbers for the Paley graphs
}

\author{
Andries E. Brouwer \\ aeb@cwi.nl
}

\author{
William J. Martin* \\ martin@wpi.edu
}

2021-09-05

\begin{abstract}
We give a tight bound for the triple intersection numbers of Paley graphs. In particular, we show that any three vertices have a common neighbor in Paley graphs of order larger than 25.
\end{abstract}

Let $q=4 t+1$ be a prime power, and let $\Gamma$ be Paley $(q)$, the Paley graph on $q$ vertices, with as vertex set the finite field $\mathbb{F}_{q}$ of size $q$, where two vertices are adjacent when their difference belongs to $\mathbb{F}_{q}^{* 2}$, the set of nonzero squares in $\mathbb{F}_{q}$. This graph is connected with diameter 2, and self-complementary.

In [5], the authors needed the fact that any function $\psi: \mathbb{F}_{q}^{* 2} \cup\{0\} \rightarrow \mathbb{C}^{*}$ satisfying (i) $\psi(0)=1$ and (ii) $\psi(a) \psi(b)=\psi(c) \psi(d)$ whenever $a+b=c+d$ must be the restriction of some additive character of $\mathbb{F}_{q}$ if $q>5$. The present note provides a short proof of that fact.

Following the notation of 2 2] $\S 3$, define generalized intersection numbers $\left[\begin{array}{cccc}a_{1} & a_{2} & \ldots & a_{\ell} \\ i_{1} & i_{2} & \ldots & i_{\ell}\end{array}\right]$ for $a_{1}, \ldots, a_{\ell} \in \mathbb{F}_{q}$ and $i_{1}, \ldots, i_{\ell} \in\{0,1,2\}$ by $\left[\begin{array}{cccc}a_{1} & a_{2} & \cdots & a_{\ell} \\ i_{1} & i_{2} & \ldots & i_{\ell}\end{array}\right]:=$ $\left|\Gamma_{i_{1}}\left(a_{1}\right) \cap \cdots \cap \Gamma_{i_{\ell}}\left(a_{\ell}\right)\right|$, where $\Gamma_{i}(a)$ denotes the set of vertices at distance $i$ from $a$. Note that $\sum_{i_{\ell}}\left[\begin{array}{lll}a_{1} & \cdots & a_{\ell} \\ i_{1} & \ldots & i_{\ell}\end{array}\right]=\left[\begin{array}{lll}a_{1} & \cdots & a_{\ell-1} \\ i_{1} & \cdots & i_{\ell-1}\end{array}\right]$ and $\left[\begin{array}{l}a \\ i\end{array}\right]=\frac{q-1}{2}$ and $\left[\begin{array}{ll}a & b \\ i & j\end{array}\right]=$ $\frac{q-1}{4}-\delta_{h i} \delta_{h j} \delta_{i j}$ for distinct $a, b$ and $h, i, j=1,2$ where $h$ is the distance from $a$ to $b$. It follows that all $\left[\begin{array}{lll}a & b & c \\ h & i & j\end{array}\right]$ are known if one knows $\left[\begin{array}{lll}a & b & c \\ 1 & 1 & 1\end{array}\right]$.

Proposition $0.1\left|\left[\begin{array}{lll}a & b & c \\ 1 & 1 & 1\end{array}\right]-\frac{q-9}{8}\right| \leq \frac{1}{4} \sqrt{q}+\frac{3}{4}$ for any three distinct $a, b, c$.

Proof. Let $\chi$ be the quadratic character. If $a, b, c$ are distinct, then

$$
\sum_{x}(1+\chi(x-a))(1+\chi(x-b))(1+\chi(x-c))=8\left[\begin{array}{ccc}
a & b & c \\
1 & 1 & 1
\end{array}\right]+4 R
$$

where $R \stackrel{x}{=}\left[\begin{array}{lll}a & b & c \\ 0 & 1 & 1\end{array}\right]+\left[\begin{array}{lll}a & b & c \\ 1 & 0 & 1\end{array}\right]+\left[\begin{array}{lll}a & b & c \\ 1 & 1 & 0\end{array}\right]$, so that $R \in\{0,1,3\}$. Let $S=\sum_{x} \chi((x-$ $a)(x-b)(x-c))$. Since $\sum_{x} 1=q$ and $\sum_{x} \chi(x)=0$ and $\sum_{x} \chi(x(x-a))=-1$ for nonzero $a$, we see that $q-3+S=8\left[\begin{array}{lll}a & b & c \\ 1 & 1 & 1\end{array}\right]+4 R$.

By Hasse [4, the number of points $N$ on an elliptic curve over $\mathbb{F}_{q}$ satisfies $|N-(q+1)| \leq 2 \sqrt{q}$. Consider the curve $y^{2}=(x-a)(x-b)(x-c)$. The homogeneous form is $Y^{2} Z=(X-a Z)(X-b Z)(X-c Z)$ with a single point $(0,1,0)$ at infinity. If $(x-a)(x-b)(x-c)$ is zero for 3 values of $x$, a nonzero square for $m$ values of $x$, and a nonsquare for the remaining $q-3-m$ values of $x$, then $N=1+3+2 m$ and $S=m-(q-3-m)=2 m+3-q$. Hence $|S|=|N-(q+1)| \leq 2 \sqrt{q}$. It follows that $\left|\left[\begin{array}{lll}a & b & c \\ 1 & 1 & 1\end{array}\right]-\frac{q-9}{8}\right| \leq \frac{1}{4} \sqrt{q}+\frac{3}{4}$.

${ }^{*}$ Worcester Polytechnic Institute, Dept. of Mathematical Sciences, Worcester, MA USA 
Corollary 0.2 If $q>25$ then any three distinct vertices in $\Gamma$ have a common neighbor.

The table below gives for small $q$ the values of $[h i j]:=\left[\begin{array}{lll}a & b & c \\ h & i & j\end{array}\right]$ that occur. For each $q$, the first line is for triangles $a b c$, the second line for paths of length 2 . The remaining cases follow by complementation.

\begin{tabular}{|c|c|c|c|c|}
\hline$q$ & {$\left[\begin{array}{lll}1 & 1 & 1\end{array}\right]$} & {$\left[\begin{array}{lll}1 & 1 & 2\end{array}\right]$} & {$\left[\begin{array}{lll}1 & 2 & 2\end{array}\right]$} & {$\left[\begin{array}{lll}2 & 2 & 2\end{array}\right]$} \\
\hline \multirow[t]{2}{*}{5} & - & - & - & - \\
\hline & 0 & 0 & 2 & 0 \\
\hline \multirow[t]{2}{*}{9} & 0 & 0 & 6 & 0 \\
\hline & 0 & 3 & 2 & 1 \\
\hline \multirow[t]{2}{*}{13} & 0 & 3 & 6 & 1 \\
\hline & $0-1$ & $3-6$ & $2-5$ & $1-2$ \\
\hline
\end{tabular}

\begin{tabular}{c|cccc}
$q$ & {$\left[\begin{array}{lll}1 & 1 & 1\end{array}\right]$} & {$\left[\begin{array}{lll}1 & 1 & 2\end{array}\right]$} & {$\left[\begin{array}{lll}1 & 2 & 2\end{array}\right]$} & {$\left[\begin{array}{lll}2 & 2 & 2\end{array}\right]$} \\
\hline 17 & 0 & 6 & 6 & 2 \\
& $1-2$ & $3-6$ & $5-8$ & $1-2$ \\
25 & $0-2$ & $6-12$ & $6-12$ & $2-4$ \\
& $2-3$ & $6-9$ & $8-11$ & $2-3$ \\
29 & 2 & 9 & 12 & 3 \\
& $2-4$ & $6-12$ & $8-14$ & $2-4$
\end{tabular}

Returning to the problem in the second paragraph, if $\psi: \mathbb{F}_{q}^{* 2} \cup\{0\} \rightarrow \mathbb{C}^{*}$ satisfies conditions (i) and (ii), then $\psi(-a)=\psi(a)^{-1}$ for each $a$ and the extension of $\psi$ to $\hat{\psi}: \mathbb{F}_{q} \rightarrow \mathbb{C}^{*}$ via $\hat{\psi}(a+b)=\psi(a) \psi(b)$ for $a, b \in \mathbb{F}_{q}^{* 2}$, is well-defined. Given $a, b \in \mathbb{F}_{q}$, we locate $c$ with $c \sim 0, a,-b$ so that $c, a-c, b+c \in \mathbb{F}_{q}^{* 2}$. Now $\hat{\psi}(a+b)=\psi(a-c) \psi(b+c)=\psi(a-c) \psi(c) \psi(-c) \psi(b+c)=\hat{\psi}(a) \hat{\psi}(b)$, showing for $q>25$ that $\hat{\psi}$ is an additive character. The cases $5<q \leq 25$ can be done by hand.

In the above, we gave bounds for $\left[\begin{array}{lll}a & b & c \\ 1 & 1 & 1\end{array}\right]$, in particular for the number of $K_{4}$ 's on a given triangle $a b c$. In case $q=p$ is prime, a closed formula for the total number of $K_{4}$ 's on a given edge was given by Evans, Pulham \& Sheehan [3]. If $p=m^{2}+n^{2}$ where $n$ is odd, this number is $\frac{1}{64}\left((p-9)^{2}-4 m^{2}\right)$.

The bounds of Proposition 0.1 are best possible:

Proposition 0.3 If $q=(4 s+1)^{2}$ for some integer $s \geq 1$, then

(i) For a suitable triangle abc one has $\left[\begin{array}{lll}a & b & c \\ 1 & 1 & 1\end{array}\right]=\frac{q-9}{8}-\frac{1}{4} \sqrt{q}-\frac{3}{4}=2\left(s^{2}-1\right)$.

(ii) For a suitable cotriangle abc one has $\left[\begin{array}{lll}a & b & c \\ 1 & 1 & 1\end{array}\right]=\frac{q-9}{8}+\frac{1}{4} \sqrt{q}+\frac{3}{4}=2 s(s+1)$.

Proof. If $a b c$ is a triangle or a cotriangle, then $\left[\begin{array}{lll}a & b & c \\ 1 & 1 & 1\end{array}\right]+\left[\begin{array}{lll}a & b & c \\ 2 & 2 & 2\end{array}\right]=\frac{q-9}{4}$. Also, $\left[\begin{array}{lll}a & b & c \\ 2 & 2 & 2\end{array}\right]=\left[\begin{array}{ccc}e a & e b & e c \\ 1 & 1 & 1\end{array}\right]$ for any nonsquare $e$. So (i) and (ii) are equivalent. Let us prove (i), that is, prove that $N=q-2 \sqrt{q}+1$ occurs for a suitable curve $y^{2}=(x-a)(x-b)(x-c)$ where $a b c$ is a triangle.

By Waterhouse [6] there are elliptic curves with $N=q \pm 2 \sqrt{q}+1$ points when $q$ is a square. A curve $y^{2}=(x-a)(x-b)(x-c)$ has three points of order 2 , so 2-torsion subgroup $\mathbb{Z}_{2} \times \mathbb{Z}_{2}$, so that its number of points is $0 \bmod 4$. Conversely, by Auer \& Top [1, given an elliptic curve $E$ with $0 \bmod 4$ points, there is one with the same number of points in Legendre form $y^{2}=x(x-1)(x-\lambda)$, except in case $q=r^{2}$ for a (possibly negative) integer $r \equiv 1(\bmod 4)$ when $|E|=(r+1)^{2}$. Consequently, there is a curve $y^{2}=x(x-1)(x-\lambda)$ with $N=(r-1)^{2}$ points. Then $S=N-(q+1)=-2 r$ and $8\left[\begin{array}{lll}0 & 1 & \lambda \\ 1 & 1 & 1\end{array}\right]+4 R=N-4=(r-1)^{2}-1=16 s^{2}-4$ and $\left[\begin{array}{lll}0 & 1 & \lambda \\ 1 & 1 & 1\end{array}\right]=2 s^{2}-\frac{R+1}{2}$. In the extreme cases, $E$ is supersingular (e.g. because $N \equiv 1(\bmod p))$ and according to [1] $(\S 3) \lambda$ is a square in $\mathbb{F}_{p^{2}}$, and then also $1-\lambda$ is a square in $\mathbb{F}_{p^{2}}$, so that $\{0,1, \lambda\}$ is a triangle and $R=3$. 


\section{Acknowledgments}

The second author thanks Bill Kantor for helpful remarks. The work of the second author was supported, in part, through a grant from the National Science Foundation (DMS Award \#1808376) which is gratefully acknowledged.

\section{References}

[1] R. Auer \& J. Top, Legendre curves over finite fields, J. Number Th. 95 (2002) 303-312.

[2] K. Coolsaet \& A. Jurišić, Using equality in the Krein conditions to prove nonexistence of certain distance-regular graphs, J. Combin. Th. (A) 115 (2008) 1086-1095.

[3] R. J. Evans, J.R. Pulham \& J. Sheehan, On the number of complete subgraphs contained in certain graphs, J. Combin. Th. (B) 30 (1981) 364-371.

[4] H. Hasse, Beweis des Analogons der Riemannschen Vermutung für die Artinschen und F. K. Schmidtschen Kongruenzzetafunktionen in gewissen elliptischen Fällen, Nachr. Ges. Wiss. Göttingen, Math.-Phys. K. (1933) 253-262.

[5] W. J. Martin and E. Washock, On ideals of the eigenpolytopes of Paley graphs and related equiangular lines, In preparation, 2021.

[6] W. C. Waterhouse, Abelian varieties over finite fields, Ann. Sci. École Norm. Sup. (4) 2 (1969) 521-560. 